\title{
The Evaluation of Tolvaptan Therapy and Long-Term Prognosis in Hepatocellular Carcinoma with Refractory Ascites
}

\author{
Masaaki Shimada*, Hiroaki Iwase, Noboru Hirashima, Masashi Saito, Hisashi Kondo, \\ Noboru Urata, Satoshi Unita, Takashi Kondo, Daiki Tanaka
}

Department of Gastroenterology, National Hospital Organization, Nagoya Medical Center, Nagoya, Japan

Email: *shimada@nnh.hosp.go.jp

How to cite this paper: Shimada, M., Iwase, H., Hirashima, N., Saito, M., Kondo, H., Urata, N., Unita, S., Kondo, T. and Tanaka, D. (2018) The Evaluation of Tolvaptan Therapy and Long-Term Prognosis in Hepatocellular Carcinoma with Refractory Ascites. Open Journal of Gastroenterology, 8, 39-44.

https://doi.org/10.4236/ojgas.2018.82004

Received: January 15, 2018

Accepted: February 10, 2018

Published: February 13, 2018

Copyright $\odot 2018$ by authors and Scientific Research Publishing Inc. This work is licensed under the Creative Commons Attribution International License (CC BY 4.0).

http://creativecommons.org/licenses/by/4.0/

\begin{abstract}
We investigated Tolvaptan efficacy and long-term prognosis with focus on nutrition in 20 patients with refractory hepatic ascites in hepatocellular carcinoma (HCC). Bloating improved in 55\% of patients, as determined using a Japanese version of the Support Team Assessment Schedule. Nutritional status improved with Tolvaptan treatment, based on the Controlling Nutritional Status score and Onodera's prognostic nutritional index. Long-term prognosis was better in responders than in non-responders (mean survival time: 308 days vs. 97 days, $\mathrm{p}=0.031$ ). Tolvaptan was even effective in many patients with HCC, with additional improvement in long-term prognosis expected with improvement in the nutritional status.
\end{abstract}

\section{Keywords}

Tolvaptan, Hepatic Ascites, Sarcopenia, Loop Diuretics, Long-Term Prognosis

\section{Introduction}

Hyponatremia, edema, and ascites related to liver cirrhosis greatly reduce the quality of life of patients [1], and furthermore, ascites worsens the prognosis in particular [2]. Malnutrition is common in patients with liver cirrhosis [3]. Tolvaptan is a new type of diuretic with vasopressin V2-receptor antagonistic action [4] [5], demonstrating an improvement in bloating in about $62.5 \%$ of patients in a Japanese phase III clinical trial [6]. According to interim results of postmarketing surveillance of Tolvaptan in liver cirrhosis (START study), the incidence of hepatocellular carcinoma (HCC) was $47.9 \%$ [7]. However, Tolvaptan therapy for HCC with refractory ascites has not been thoroughly studied. 
Therefore, we evaluated the outcome of Tolvaptan therapy and long-term prognosis in HCC patients with refractory ascites, focusing particularly on the nutritional status.

\section{Materials and Methods}

We retrospectively studied the data of 20 patients with refractory hepatic ascites in HCC who did not respond adequately to conventional diuretics treatment with a loop diuretic and/or an anti-aldosterone agent between April 2011 and March 2017 with Tolvaptan. HCC was diagnosed based on dynamic computed tomography or magnetic resonance imaging.

The degree of bloating was determined before and after 3 weeks of Tolvaptan therapy using the Japanese version of the Support Team Assessment Schedule (STAS-J) [8]. STAS-J is scored from 0 to 4 as follows; 0 : there is no symptom. 1) there are occasional or intermittent single or several symptoms at a time, but the patients can continue with everyday life and their symptoms do not require treatment; 2) there are symptoms of a moderate degree on days when the patient's condition is poor, and it may affect the ability to perform activities of daily living; 3$)$ there are often strong symptoms, and they remarkably affect concentration and ability to perform activities of daily living; and 4) there are continuous, intolerable, and intense symptoms. The patient cannot think about other things. A patient was defined as a responder when the score decreased by 1 point or more after Tolvaptan administration. Furthermore, the efficacy rate of Tolvaptan on a decrease in the body weight of more than $1.5 \mathrm{~kg}$ [9] was evaluated.

Nutritional status was estimated by caloric intake, controlling nutritional status (CONUT) method [10], and Onodera's prognostic nutritional index (PNI) [11]. Long-term prognosis was evaluated after Tolvaptan therapy administration.

As for a retrospective study, missing data were not available. Body weight on day 7 was not measured in seven patients, and CONUT score was not calculated in 3 patients. Statistical differences were analyzed using Wilcoxon signed-ranks test. The long-term prognosis was estimated using the Kaplan-Meier method and differences were determined using the log-rank test. $P$-values $<0.05$ were considered statistically significant.

This study was approved by the ethical review board of the Nagoya Medical Center.

\section{Results}

There were 9 men and 11 women, with an average age of $72.6 \pm 10.6$ years. The etiology of liver cirrhosis was as follows: HBV, 2 cases; HCV, 15 cases; alcoholic, 1 case; and non-alcoholic fatty liver disease, 2 cases. HCC stage-wise break up was stage I, 5 cases; II, 3 cases; III, 3 cases; and IV, 9 cases [12]. According to the Child-Pugh classification [13], 9 cases were classified as class B and 11 cases as class $\mathrm{C}$, and the mean Child-Pugh score was $10.1 \pm 1.9$ (Table 1). 
Table 1. Baseline characteristics of patients.

\begin{tabular}{cc}
\hline Age (years) & $72.6 \pm 10.6$ \\
Gender (male/female) & $9 / 11$ \\
Bodyweight $(\mathrm{kg})$ & $57.9 \pm 10.6$ \\
BMI $\left(\mathrm{kg} / \mathrm{m}^{2}\right)$ & $23.7 \pm 4.9$ \\
Etiology of chronic liver disease & \\
Hepatitis B/Hepatitis C/Alcoholic/NAFLD & $2 / 15 / 1 / 2$ \\
HCC stage I/II/III/IV & $5 / 3 / 3 / 9$ \\
Child-Pugh classification (B/C) & $9 / 11$ \\
Child-Pugh score & $10.1 \pm 1.9$ \\
STAS-J score & $3.3 \pm 0.7$ \\
Caloric intake (kcal) & $799 \pm 376$ \\
Alb $(\mathrm{g} / \mathrm{dL})$ & $2.5 \pm 0.5$ \\
TLC $(/ \mu \mathrm{L})$ & $759 \pm 522$ \\
T-cho $(\mathrm{mg} / \mathrm{dL})$ & $115 \pm 33$ \\
CONUT score & $8.3 \pm 2.4$ \\
\hline
\end{tabular}

Data are expressed as number or mean \pm SD. BMI: body mass index, NAFLD: non-alcoholic fatty liver disease, HCC: hepatocellular carcinoma, STAS-J: Japanese version of the Support Team Assessment Schedule, Alb: albumin, TLC: total lymphocyte count, T-cho: total cholesterol, CONUT: controlling nutritional status.

The patients' mean STAS-J score significantly decreased from $3.3 \pm 0.7$ to $2.4 \pm$ $1.1(P<0.001$, response rate: $55 \%)$, and the decrease in body weight more than $1.5 \mathrm{~kg}$ was $69.2 \%$ after Tolvaptan administration.

The caloric intake increased from $799 \pm 376 \mathrm{kcal}$ to $1073 \pm 478 \mathrm{kcal}(P=$ $0.025)$, and the CONUT score improved from $8.3 \pm 2.4$ to $7.7 \pm 2.9(P=0.016)$ after administration of Tolvaptan.

Onodera's PNI did not show improvement in non-responders (from $26.0 \pm$ 4.7 to $25.6 \pm 5.6, P=0.286$ ), whereas it revealed improvement in responders (from $23.9 \pm 5.7$ to $28.1 \pm 5.1, P=0.009$ ).

The mean survival time (MST) was 217 days (Figure 1(a)). Long-term prognosis improved in responders compared with non-responders (MST: 308 days vs. 97 days, $P=0.031$ ) (Figure 1(b)).

\section{Discussion}

Improvement in bloating was evaluated using STAS-J scores in our previous report [14]. The STAS-J score [8] was improved in 55\% of the patients with refractory hepatic ascites in HCC who did not respond adequately to conventional diuretics. It was reported that the efficacy was judged by a decrease in the body weight of more than $1.5 \mathrm{~kg}$ after one week of Tolvaptan administration in 2017, and we judged the efficacy of our patients, with the same criteria, the response rate being $69.2 \%$. These results revealed that Tolvaptan therapy was effective even in patients with HCC.

The CONUT method [10] uses three blood measurements, serum albumin, 


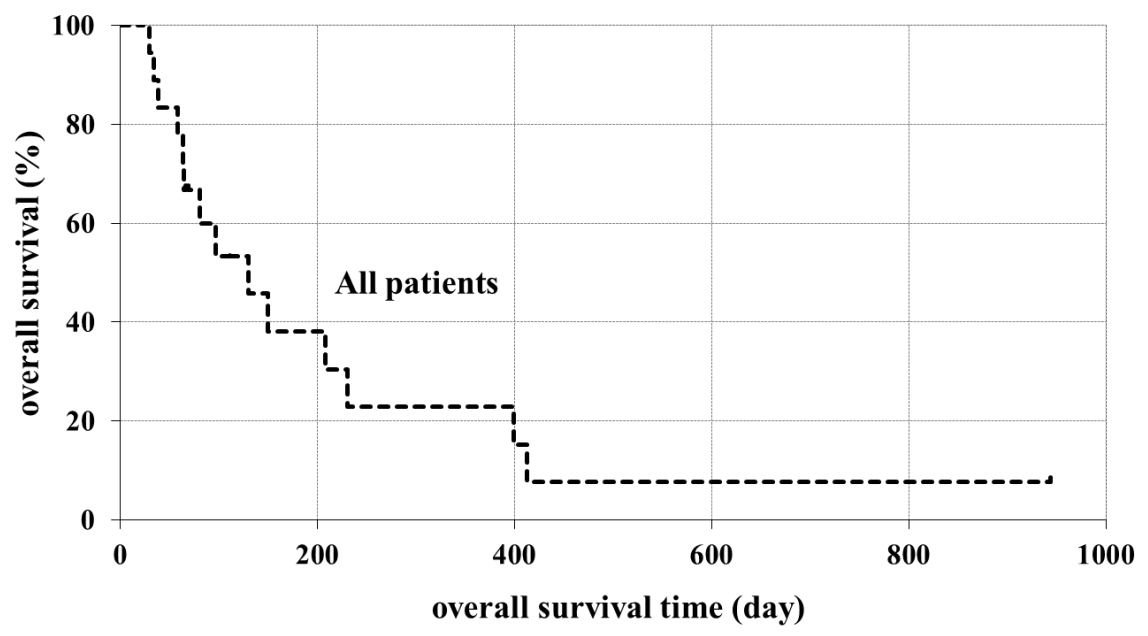

(a)

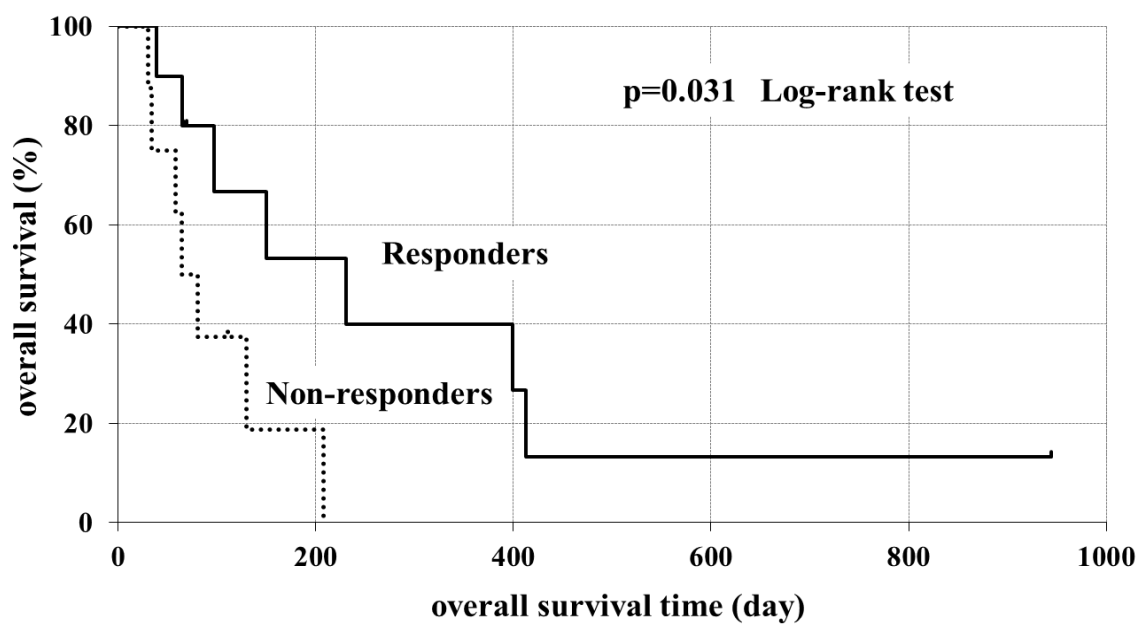

(b)

Figure 1. Long term prognosis. (a) All patients; (b) Compared with responders and non-responders.

total lymphocyte count, and total cholesterol level, and it classifies nutritional status into four categories: normal (0 - 1), low (2 - 4), moderate (5 - 8), and severe $(9-12)$. Malnutrition was present in $63 \%$ of patients with liver cirrhosis, using the CONUT method [15].

Majority of the patients had moderate or severe malnutrition according to the CONUT score $(8.3 \pm 2.4)$ before Tolvaptan induction. An improvement in bloating was achieved after Tolvaptan induction, and the caloric intake increased. Furthermore, the CONUT score decreased significantly, so the nutritional status improved.

Onodera's PNI [11] is a malnutrition state rating system to predict operative risk. It was calculated considering two factors, serum albumin level and total lymphocyte counts, the reliability being high for the evaluation of postoperative condition and patient's prognosis.

There was significant improvement of Onodera's PNI in the responders group 
before and after Tolvaptan therapy, but not in the non-responders group. These results suggested that long-term prognosis was dependent on the improvement of the patient's nutritional status.

Patients with refractory hepatic ascites in HCC have poor nutritional status, and are diagnosed as being in hepatic failure, thus negating any aggressive treatment options for HCC. Refractory hepatic ascites decreased or disappeared, and nutritional status improved in many cases in this study, so it is expected that patients will receive aggressive treatment in the future. Furthermore, Tolvaptan therapy will contribute to the improvement of long-term prognosis.

This was a retrospective study in a single institution, and in future, a larger, multicenter prospective study should be performed to confirm our findings.

\section{Conclusion}

In conclusion, Tolvaptan therapy was effective in several patients with refractory hepatic ascites in HCC, demonstrating additional improvement in long-term prognosis due to an improvement in the nutritional status.

\section{References}

[1] Sola, E., Watson, H., Graupera, .I, et al. (2012) Factors Related to Quality of Life in Patients with Cirrhosis and Ascites: Relevance of Serum Sodium Concentration and Leg Edema. Journal of Hepatology, 57, 1199-1206. https://doi.org/10.1016/j.jhep.2012.07.020

[2] Llach, J., Gines, P., Arroyo, V., et al. (1988) Prognostic Value of Arterial Pressure, Endogenous Vasoactive Systems, and Renal Function in Cirrhotic Patients Admitted to the Hospital for the Treatment of Ascites. Gastroenterology, 94, 482-487. https://doi.org/10.1016/0016-5085(88)90441-6

[3] Larramona, G.L., Lucendo, A.J. and Tenias, J.M. (2015) Association between Nutritional Screening via the Controlling Nutritional Status Index and Bone Mineral Density in Chronic Liver Disease of Various Etiologies. Hepatology Research, 45, 618-628. https://doi.org/10.1111/hepr.12395

[4] Yamamura, Y., Nakamura, S., Itoh, S., et al. (1998) OPC-41061, a Highly Potent human Vasopressin V2-Receptor Antagonist: Pharmacological Profile and Aquaretic Effect by Single and Multiple Oral Dosing in Rats. Journal of Pharmacology and Experimental Therapeutics, 287, 860-867.

[5] Ohki, T., Sato, K., Yamada, T., et al. (2015) Efficacy of Tolvaptan in Patients with Refractory Ascites in a Clinical Setting. World Journal of Hepatology, 7, 1685-1693. https://doi.org/10.4254/wjh.v7.i12.1685

[6] Sakaida, I., Kawazoe, S., Kajimura, K., et al. (2014) Tolvaptan for Improvement of Hepatic Edema: A Phase 3, Multicenter, Randomized, Double-Blind, Placebo-Controlled Trial. Hepatology Research, 44, 73-82. https://doi.org/10.1111/hepr.12098

[7] Sakaida, I., Terai, S., Kurosaki, M., et al. (2017) Effectiveness and Safety of Tolvaptan in Liver Cirrhosis Patients with Edema: Interim Results of Post-Marketing Surveillance of Tolvaptan in Liver Cirrhosis (START Study). Hepatology Research, 47, 1137-1146. https://doi.org/10.1111/hepr.12852

[8] Miyashita, M., Matoba, K., Sasahara, T., et al. (2004) Reliability and Validity of Japanese Version STAS (STAS-J). Palliative and Supportive Care, 2, 379-384. https://doi.org/10.1017/S1478951504040507 
[9] Hiramine, Y., Uojima, H., Nakanishi, H., et al. (2017) Response Criteria of Tolvap$\tan$ for the Treatment of Hepatic Edema. Journal of Gastroenterology.

[10] Ignacio de Ulibarri, J., Gonzalez-Madrono, A., de Villar, N.G., et al. (2005) CONUT: A Tool for Controlling Nutritional Status. First Validation in a Hospital Population. Nutricion Hospitalaria, 20, 38-45.

[11] Onodera, T., Goseki, N. and Kosaki, G. (1984) Prognostic Nutritional Index in Gastrointestinal surgery of Malnourished Cancer Patients. Nihon Geka Gakkai Zasshi, 85, 1001-1005.

[12] The Liver Cancer Study Group of Japan Society of Hepatology (2015) The General Rules for the Clinical and Pathological Study of Primary Liver Cancer. 6th Edition, Kanehara \& Co Ltd., Tokyo.

[13] Pugh, R.N.H., Murray-Lyon, I.M., Dawson, J.L., et al. (1973) Transection of the Oesophagus for Bleeding Oesophageal Varices. British Journal of Surgery, 60, 646649. https://doi.org/10.1002/bjs. 1800600817

[14] Shimada, M., Iwase, H., Hirashima, N., et al. (2017) Nutritional Status and LongTerm Prognosis in Patients with Refractory Hepatic Ascites Treated with Tolvaptan. Journal of Gastroenterology, Hepatology and Endoscopy, 2, 1-5.

https://doi.org/10.15761/GHE.1000131

[15] Larramona, G.L., Lucendo, A.J. and Tenias, J.M. (2015) Association between Nutritional Screening via the Controlling Nutritional Status Index and Bone Mineral Density in Chronic Liver Disease of Various Etiologies. Hepatology Research, 45, 618-628. https://doi.org/10.1111/hepr.12395 